\title{
Study of the relationship between sphenoid sinus volume and protrusions in the sphenoid sinus
}

\author{
Yuefeng $\mathrm{Li}^{1,2}$, Jiaqi Sun ${ }^{1,2}$, Xiwen $\mathrm{Zhu}^{3}$, Chenhao Zhao ${ }^{4}$, Jin $\mathrm{Xu}^{1}$, \\ Ping Jiang ${ }^{1^{*}}$, Xinkang Tong ${ }^{1}$ \\ ${ }^{1}$ Department of Clinical and Imaging Anatomy, School of Medicine Science and Laboratory Medicine, Jiangsu University, Zhenjiang, \\ China; ${ }^{*}$ Corresponding Author: jiangping@ujs.edu.cn \\ ${ }^{2}$ Department of Medical Imaging, Affiliated Hospital of Jiangsu University, Zhenjiang, China \\ ${ }^{3}$ Department of Otolaryngology, Affiliated Hospital of Jiangsu University, Zhenjiang, China \\ ${ }^{4}$ Department of Ophthalmology, Fourth Affiliated Hospital of Jiangsu University, Zhenjiang, China
}

Received 14 September 2013; revised 29 October 2013; accepted 8 November 2013

Copyright (C) 2014 Yuefeng Li et al. This is an open access article distributed under the Creative Commons Attribution License, which permits unrestricted use, distribution, and reproduction in any medium, provided the original work is properly cited. In accordance of the Creative Commons Attribution License all Copyrights (C) 2014 are reserved for SCIRP and the owner of the intellectual property Yuefeng Li et al. All Copyright @ 2014 are guarded by law and by SCIRP as a guardian.

\section{ABSTRACT}

The relationship between the volume of sphenoid sinus (SS) and the prevalence of internal carotid artery (ICA) and optic nerve (ON) protrusions in the SS was studied by using high-resolution CT imaging. The ICA and ON protrusions in SS were observed in randomly selected normal head CT scanning images from 350 adult subjects. According to the incidence of ICA protrusion, three groups were divided into no ICA protrusion (70.75\%), unilateral protrusion (8.68\%) and bilateral protrusions $(20.57 \%)$. The ON protrusion accounted for $16 \%$ in 350 subjects and accompanied absolutely with ICA protrusion, but ICA protrusion appeared without accompanying with ON protrusion. The SS volume depended upon the protrusions in it and showed statistical differences, without ICA protrusion, the smallest size $(11.16 \pm 1.60) \mathrm{cm}^{3}$; the unilateral protrusion, medium size $(14.20 \pm 1.80) \mathrm{cm}^{3}$ and the bilateral protrusion, the largest size $(25.03 \pm 2.21) \mathrm{cm}^{3}$. By observing 3D reconstructed models of $\mathrm{ON}$ and SS, we found ON was adjacent to SS (46\%) and to posterior ethmoid sinuses (44\%). The current study indicates that SS volume is varied with numbers of the protrusions and that ON location varies with the pneumatization of SS. Our results provide an anatomical basis to the surgeries for SS and its surrounding structures.

\section{KEYWORDS}

\section{Sphenoid Sinus Volume; Protrusion of Internal}

\section{Carotid Artery; CT Imaging}

\section{INTRODUCTION}

With the advance of the endoscopic endonasal transsphenoidal approach, it is possible to make it instead of removal of pituitary by trans-skull cavity, not only for endoscopic pituitary via the sphenoid sinus, but also for some operations needed to be expand to sellar region, suprasellar lesions, cavernous sinus, clivus and et al. SS is surrounded by important structures, such as, internal carotid artery (ICA), optic nerve (ON), et al. [1]. Therefore, blindness or massive bleeding and even minimal damage to surrounding structures during an operation can lead to an irrevocable outcome [2]. Several important surgical landmarks, such as recesses, prominences, and impressions caused by neurovascular structures coursing underneath the bone, could be clues to locate these vital structures, which help avoid injury during the operation. Anatomical and radiological studies dealt with the sphenoid sinus and its surrounding structures based on cadavers or CT studies in these years. Several literatures have documented the dimensions, septation, pattern of SS pneumatization, SS surrounding structures [3-5], prevalence of protrusions [6] and the relation between pneumatization and protrusions [7]. These studies provided important information for surgeries in this area and promoted advancement of the surgery as well. In the current study, we accomplished volumetric measurement of SS by using 3D image reconstruction based on conventional two-dimensional CT images and the types were divided according to the numbers of ICA protrusion in SS, and the relation between the number of the ICA pro- 
trusion and SS volume was analyzed. Furthermore, the relation between spatial position of SS and its adjacent important structures in 3-dimension model was observed in order to prevent intraoperative complicating.

\section{MATERIALS AND METHODS}

Approval for this study was obtained by the ethical committee of affiliated hospital of Jiangsu university. CT scan images from 350 Chinese subjects with no obvious abnormalities in paranasal sinuses by Spiral CT were collected in Department of Otolaryngology_-Head and Neck Surgery, Jiangsu University Affiliated Hospital from January 2009 to March 2012. Of the subjects, 188 were males and 162 females, age ranged between 25 and 64. The subjects were scanned on GE 64-row 128-slice VCT according to the following parameters: slice thickness $-3.75 \mathrm{~mm}$, increment $-1 \mathrm{~mm}, 120 \mathrm{kV}, 230-280$ $\mathrm{mA}$, Collimator width $3-5 \mathrm{~cm}$, Scan type-Helical full $1.0 \mathrm{sec}$ FOV: 25.0, Bone window (center $800 \mathrm{HU}$, width $2000 \mathrm{HU}$ ). Taking the hard palate as reference axis, in the coronal study the plane of section was perpendicular to this structure. Axial sections were performed in a plane parallel to the hard palate from the upper dental arch to the roof of the frontal sinuses. Data acquired on the axial plane and coronal plane were created by secondary reconstruction method in the workstation. The thickness of reconstructed image slice was of $-1 \mathrm{~mm}$. 3D reconstruction from these slices was established with the MIMICS software.

The ON and ICA protrusions in the SS were observed on the axial and coronal plane on personal computers. Firstly, the presence of the ICA and ON protrusions was identified and assessed on the axial plane according to Unal's method [6]. Briefly, the protrusion of the ICA was determined as finding for placement of ICA with 50\% of its diameter in SS and obvious or light protrusions was determined as finding for placement of ICA with more than $50 \%$ or $10 \%-50 \%$ of its diameter. Presence of air and density around $\mathrm{ON}$ was accepted as a clue of $\mathrm{ON}$ protrusion. Then, one or double or no protrusions in the SS were asserted with head CT scan imaging and the location of ON which passed caudally from the orbit and connected the optic chiasm were searched with the 3D model of SS and its nearby structures done by MIMICS. The relationship between the ON and the SS was also evaluated.

The presence of the ICA and ON protrusions in the SS was observed on the axial and coronal plane of head CT scan imaging of 350 cases. According to whether the ICA protrusions existence, 3 groups were divided and named as NO protrusion of ICA, one protrusion of ICA and double protrusions of ICA. Furthermore, 90 cases (3 groups, each group contained 30 subjects) were random- ly selected and analyzed by a software, MIMICS 12.0, the Belgian Materialise's three-dimensional medical image processing to outline SS, ICA and ON. The results were submitted to the statistical correlation study between the SS volume and the ICA protrusions. The threshold value was ensured before the outlining and its value was: Min -1024, Max -129. SS on each slice was drawn by semi-automatic method, but ON and ICA were drawn manually. The volume of SS was calculated by the MIMICS software. Three-dimensional models of SS, ICA and ON from 100 randomly selected cases were subsequently established and their spatial position were observed under the $3 \mathrm{D}$ reconstruction for the relationship between $\mathrm{ON}$ with SS. In order to reduce possible errors, all images were observed and outlined by three independent researchers. Data obtained from these two groups were statistically determined using Kappa coefficient determinant and Chi-square analysis. All calculations were performed using the SPSS package program 11.5. Data are expressed as mean \pm standard deviation and were analyzed by one-way ANOVA, p values $<0.05$ was statistically significant.

\section{RESULTS}

\subsection{Characteristics of Protrusions in the SS}

According to the morphological feature, the protrusions into SS were divided to three types when the SS was observed in the axial plane (Figure 1) on the CT scan images from 350 subjects (Figure 1(A)), no ICA protrusion the in the SS (247 subjects, 70.75\%), unilateral protrusion (31 subjects, $8.68 \%$, Figure $1(\mathrm{~B})$ ), and bilateral protrusions (72 subjects, 20.57\%, Figure 1(D)). Further, ICA protrusion in the SS, with a dehiscence of bony wall of the ICA (Figure 1(D)) was found in 8 subjects. We also observed ON protrusion on the coronal and axial planes by using same head CT scan imaging described above. An ON protrusion was seen in 56 subjects (16\%), in which 54 cases were bilateral and only 2, unilateral (Figure 2(A)). ON exposed in SS cavity was found in 7 cases because of the dehiscence of bony wall. Pneumatization of anterior clinoid process (ACP) was happened in 35 cases with ON process from 350 subjects (10\%, including 4\% bilateral and $6 \%$ unilateral). The ACP cavity with pneumatization became bigger in 24 sides because they were fused to the SS (Figure 2(D)). ON located and passed in SS because of existence of ACP. ON was found passing freely in the posterior ethmoid sinus on 21 sides because ACP fused with the posterior ethmoid sinus. The relationship between protrusion of the ON and ICA showed that ON protrusions accompanied absolutely with ICA protrusion, however, ICA protrusion didn't always accompany with ON protrusion. 

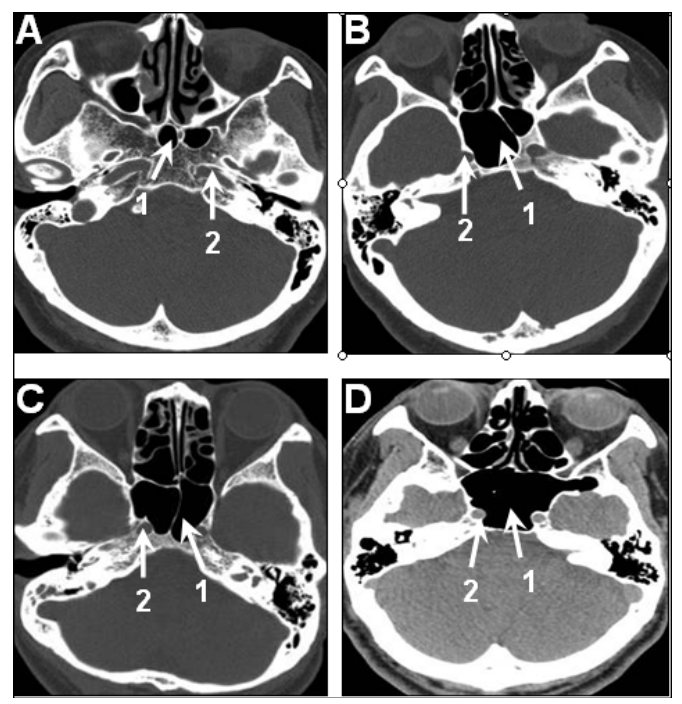

Figure 1. The type of the protrusion in the sphenoid sinus. NO protrusion in the sphenoid sinus and poor pneumatization sphenoid sinus (A). Unilateral protrusion of ICA in the SS and right ICA is bulged into the SS slightly. The area of SS is large (B). Bilateral protrusion of ICA in the SS and right ICA is bulged into the SS clearly and the area of SS becomes larger (C). Bilateral protrusion of ICA in the SS and bilateral ICA is bulged into the SS clearly and there is a dehiscence of bony wall of right ICA. The area of SS becomes much larger. 1. Sphenoid sinus (SS); 2. Internal carotid artery (ICA).

\subsection{The Relevance between Prevalence of ICA Protrusions and SS Volume}

According to the prevalence of ICA protrusions in SS, three groups were divided: no protrusion, unilateral protrusion and bilateral protrusions. 30 subjects were randomly selected from each group for measuring SS volume and analyzing relationship between protrusion and volume. The results showed the smallest SS volume existed in the cases without protrusion but the largest volume with bilateral protrusions (Figure 3).

\subsection{The Spatial Position of ON}

The relation between ON and SS was observed on the 3D reconstruction model of SS and its surrounding structures by MIMICS (Figure 4). The ON leaves the orbital cavity via the optic canal and passes through the lateral wall of the posterior ethmoid sinus and SS, and then, it passes posterior-medially form the optic chiasm. We selected randomly 100 subjects from 350 cases for analysis and found three types: Type 1 (46 subjects, $46 \%)$, ON lay on the superior of the lateral wall of SS (Figure 2(A)), its main anatomical characteristic was the ON contacting the superior part of SS; Type 2 (44 subjects, 44\%) with ON passing through the lateral wall of the ethmoid sinus and anterolateral corner of SS (Fig- ures 2(B) and (C)), then running caudally to superior ending at the optic chiasm, and its main anatomical feature was minimal contacting area between the superior part of the SS and the ON; Type 3 (10 subjects,10\%) showed pneumatization of ACP. The cavity of pneumatization was fused with the SS or ethmoid sinus and ON was passed in the SS or ethmoid sinus (Figure 2(D)). The ON located at the superior part of the lateral wall of SS on triplanar images. However, ON has its different position if viewing from different plane, ON located at lateral-superior part of the SS on the coronal plane, anterior edge of superior part of the SS on the sagittal plane and lateral wall of the SS on the axial plane (Figure 5). On the 3D models, more detail morphological features were showed that pneumatization cavity of the ACP was fused with SS, ON passed through the SS cavity (Figure 4(A)) and ON was bulged into the SS (Figure 4(B)).

\section{DISCUSSION}

\subsection{About Experimental Design}

We used two novel experimental methods in the present study. According to ICA protrusion (with or not or one or two) in the SS, the volume of SS was divided into three types: type 1 , no protrusion in the SS, type 2, unilateral protrusion in the SS, and type 3, bilateral pro-
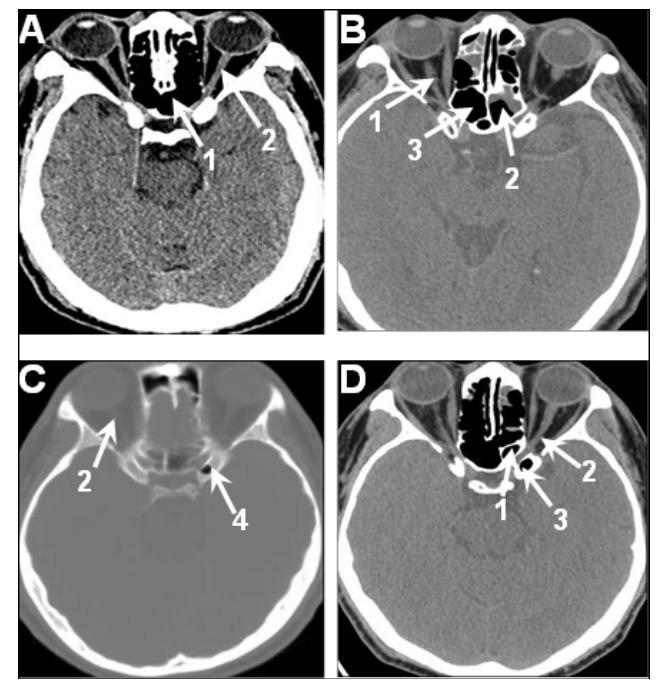

Figure 2. The types of optic nerve near the sphenoid sinus. Optic nerve (ON) is running at the lateral wall of SS and nudity (A). The position of optic nerve, the right ON locates on the lateral wall of ethmoid sinus and left on the lateral wall of SS (B), 1. ON; 2. SS; 3. Ethmoid sinus. The position of ON (ON locates on the above of SS after it passes through lateral wall of ethmoid sinus) (C), 2. ON; 4. Pneumatization cavity of ACP. Pneumatization of ACP (ON runs across between SS and pneumatization cavity of ACP when left pneumatization of ACP) (D), 1. SS; 2. ON; 3. Pneumatization cavity of ACP. 

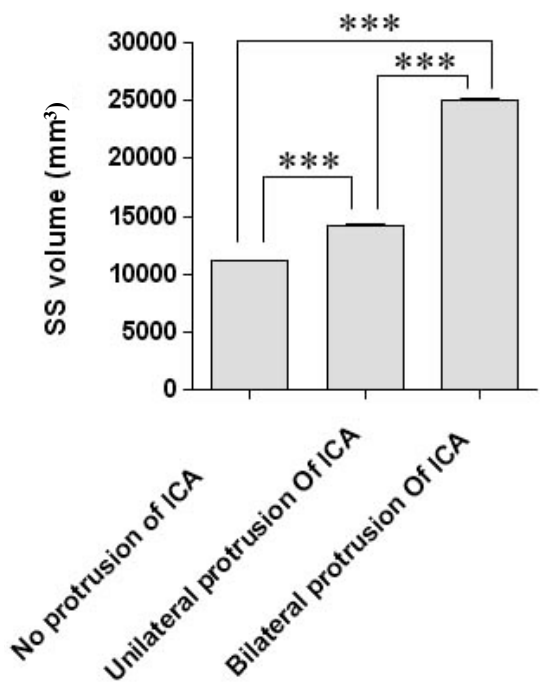

Figure 3. The relation of prevalence between ICA protrusions and SS volume. The SS volumes from three groups were analyzed by one-way ANOVA, ${ }^{* * *} \mathrm{p}<0.001$ for no protrusion vs unilateral protrusion or bilateral protrusions.
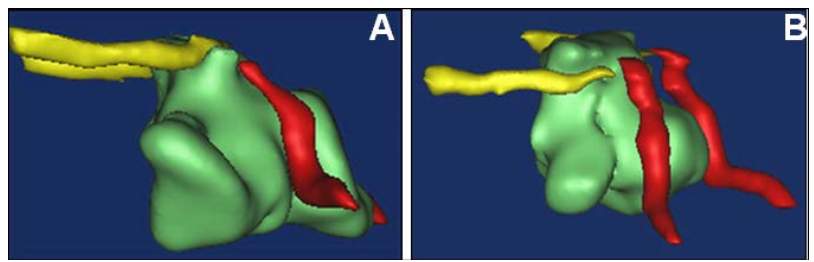

Figure 4. 3D reconstruction of SS and its surrounding structures made by MIMICCS. It shows left side of 3D reconstruction of SS. SS is green, ON in yellow and ICA in red. Pneumatization of left ACP was fused with SS and formed a large cavity of SS and ON runs across between SS and pneumatization cavity of ACP and ON locates in the SS (A). ON and ICA was bulged into the SS and formed to ON and ICA protrusions (B) in the SS.
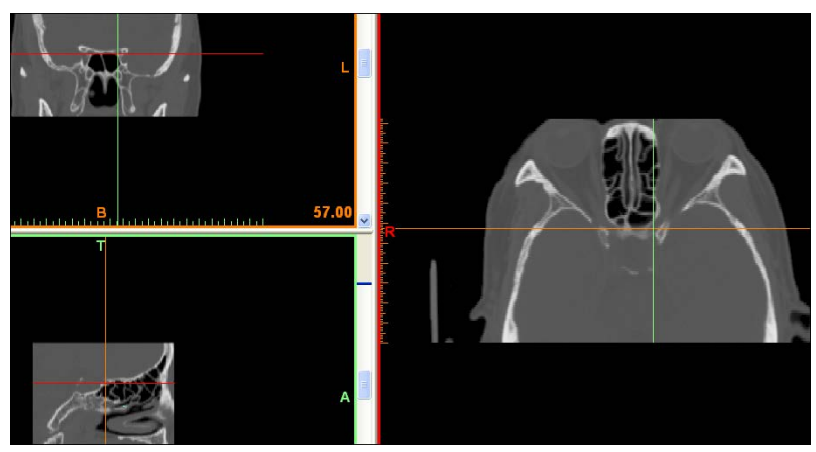

Figure 5. Triplanar images of SS and ON formed by MIMICCS. The top image on the left panel showed coronal plane and bottom sagittal plane. Right panel showed axial plane. The $\mathrm{ON}$ is indicated by the point crossing with two lines. ON is located lateral-superior at the SS on the coronal plane and superior at the SS on the sagittal plane and lateral wall at the SS on the axial plane. trusion in the SS. We came up with the classification so that we can predict whether to carry operative procedure or evaluate risky factor of anatomic variations of SS during operation according to the SS volume. The model of 3D reconstruction of SS and its surrounding structures were made with MIMICS software and got SS volume parameters automatically. To our knowledge, the current study explored for the first time to show these structures in three dimensions and there has been considerable interest in rendering 2D images into 3D images that more closely mimic the surgical environment of the sellar area. Image reconstruction of normal anatomical structures has been employed for surgical training and preoperative planning, including transsphenoidal surgery [2].

\subsection{The Relationship between SS Volume and Transsphenoidal Surgical Approach}

The endoscopic endonasal transsphenoidal approach was proposed in the past decade as a minimally invasive surgical technique and gained significant popularity because some structures around SS might be carried by SS with endoscopy surgery, for example pituitary surgery. Neighboring structures, such as protrusions of ICA, ON and maxillary nerve are bulged into the SS so that proportional protrusions can be seen in the SS with endoscopy. These special bulges can also be used as markers when transsphenoidal endoscopic surgery is carried out. However, the protrusions don't appear in all cases. The prevalence of the protrusions is quite variable, which may affect the success of transsphenoidal endoscopic surgery [6,8-13]. The prevalence of protrusion of ICA is not conclusive, ranging from 5.2\% [14] - 32.8\% [15], $48.5 \%$ [16] and $67.7 \%$ [8], depending on the degree of individual pneumatization and the definition used in each study. From these results, the distances between these structures and the wall of SS and the prevalence of ptotrusions in SS were found proportion to the degree of SS pneumatization [7], but the quantitative evaluation SS volume was not indicated. Therefore, a question was raised: do any rules of the prevalence of protrusions exist in the SS [17]? In the present study, we have researched the relationship between the prevalence of protrusion and volumes of SS by measuring the images of head CT with software and it showed that there is a highly statistically significant difference between the prevalence of protrusion and volumes of SS. There was no protrusion as the SS volume was small $\left(1156 \mathrm{~mm}^{3}\right)$. However, with the SS volume increased, a protrusion appeared first and then up to two protrusions appeared. Therefore, we consider that the prevalence of protrusion is proportionally related to the SS volume or size; the larger the volume, the higher the prevalence, and vise verse. Our results have showed that there is a significant associated relationship between 
prevalence of protrusion of ICA and ON in the SS, and the appearance $\mathrm{ON}$ protrusion always accompanies with ICA protrusion, however, the appearance of ICA protrusion may not accompany with ON. It is implied that injure could be avoided during optic nerve decompression if an ICA protrusion in the SS should be thought. We suggest that preoperative CT examination of the SS is extremely helpful in identifying the pneumatization degree of SS, analyzing the pneumatization type, distinguishing relation with other important structures and defining the degree of bulges in the SS. In the event that the SS is low pneumatization so that the ICA and ON do not bulge to the sinus cavity, and an endoscopic transsphenoidal operation might not be the first choice. If SS is high pneumatization with protrusions of the ICA and ON, the surgeons might make some surgeries such as carotid aneurysm and optic nerve decompression. Obviously, the protrusions in the SS are related to SS volume highly, and the size of volume of the SS is also a key for the success of endoscopy with transsphenoidal surgery.

\subsection{The Relation between SS and ON and Its Clinical Anatomy}

The volume of SS has to be determined before transsphenoidal approach. If there are some protrusions in the SS or pneumatization of ACP, the volume of SS should be larger, which makes people easy to identify and visualize nearby structures of SS and adapt to surgery [17-19]. When you do ON decompression, you should take into account that there is an ICA protrusion except for ON protrusion in the SS because ICA protrusion usually accompanies with ON protrusion and pays attention to protect ICA and other structures during surgery. In the present study, almost $50 \%$ of ACP pneumatized cavities were fused with the posterior ethmoid sinus, and $\mathrm{ON}$ ran in the posterior ethmoid sinus freely or located at the lateral wall of the posterior ethmoid sinus. Therefore, it is difficult to find ON if transsphenoidal approach is selected because ON ran in the ethmoid sinus rather than located in SS.

Two types of relation between ON and SS were found in current study. One was that ON located on the superior and lateral wall of the SS, and another one was that ON located at the anterior and lateral corner of SS. For the former, surgery is advised to be carried out because of good pneumatization of SS. During the operation, ON can not only be identified at the anterior and lateral horn of SS, but also be found at the superior and lateral wall of SS. By this way, the operative field of the $\mathrm{ON}$ is broadened to benefit the surgery. The ON decompression in SS is not advised if the patient is the type of ON locating at the anterior and lateral corner of SS and ON passing along the lateral wall of ethmoid sinus, so that contacting area of ON to SS becomes very small. It is difficult to find ON if transsphenoidal approach is selected because ON runs in the ethmoid sinus rather than locates in the SS, and therefore, the key is to identify if ON locates in SS or in ethmoid sinus before surgery.

\section{REFERENCES}

[1] Edem, I.J., Banton, B., Bernstein, M., Lwu, S., et al. (2013) A prospective qualitative study on patients' perceptions of endoscopic endonasal transsphenoidal surgery. British Journal of Neurosurgery, 27, 50-55.

http://dx.doi.org/10.3109/02688697.2012.711497

[2] Wang, S.S., Xue, L., Jing, J.J. and Wang, R.M. (2012) Virtual reality surgical anatomy of the sphenoid sinus and adjacent structures by the transnasal approach. Journal of Cranio-Maxillofacial Surgery, 40, 494-499.

http://dx.doi.org/10.1016/j.jcms.2011.08.008

[3] Zada, G., Agarwalla, P.K., Mukundan, S., et al. (2011) The neurosurgical anatomy of the sphenoid sinus and sellar floor in endoscopic transsphenoidal surgery. Journal of Neurosurgery, 114, 1319-1330.

[4] Lu, Y., Pan, J., Qi, S., et al. (2011) Pneumatization of the sphenoid sinus in Chinese: The differences from causian and its application in the extended transsphenoidal approach. Journal of Anatomy, 219, 132-142. http://dx.doi.org/10.1111/j.1469-7580.2011.01380.x

[5] Ramos, H.F., Monteiro, T.A., Pinheiro Neto, C.D., et al. (2011) Endoscopic anatomy of the approaches to the sellar area and planum sphenoidale. Arquivos de Neuropsiquiatria, 69, 232-236. http://dx.doi.org/10.1590/S0004-282X2011000200018

[6] Unal, B., Bademci, G., Bilgili, Y.K., et al. (2006) Risky anatomic variations of sphenoid sinus for surgery. Surgical and Radiologic Anatomy, 28, 195-201. http://dx.doi.org/10.1007/s00276-005-0073-9

[7] Güldner, C., Pistorius, S.M., Diogo, I., et al. (2012) Analysis of pneumatization and neurovascular structures of the sphenoid sinus using cone-beam tomography (CBT). Acta Radiologica, 53, 214-219. http://dx.doi.org/10.1258/ar.2011.110381

[8] Tan, H.K. and Ong, Y.K. (2007) Sphenoid sinus: An anatomic and endoscopic study in Asian cadavers. Clinical Anatomy, 20, 745-750. http://dx.doi.org/10.1002/ca.20507

[9] Pirner, S., Tingelhoff, K., Wagner, I., et al. (2009) CTbased manual segmentation and evaluation of paranasal sinuses. European Archives of Oto-Rhino-Laryngology, 266, 507-518. http://dx.doi.org/10.1007/s00405-008-0777-7

[10] Kim, J., Song, S.W., Cho, J.H., et al. (2010) Comparative study of the pneumatization of the mastoid air cells and paranasal sinuses using three-dimensional reconstruction of computed tomography scans. Surgical and Radiologic Anatomy, 32, 593-599. http://dx.doi.org/10.1007/s00276-009-0618-4

[11] Emirzeoglu, M., Sahin, B., Bilgic, S., et al. (2007) Volu- 
metric evaluation of the paranasal sinuses in normal subjects using computer tomography images: A stereological study. Auris Nasus Larynx, 34, 191-195. http://dx.doi.org/10.1016/j.anl.2006.09.003

[12] Anta Escuredo, J.A., Sánchez Del Rey, A., et al. (2000) Morphometric study of the paranasal sinuses in normal and pathological conditions. Acta Oto-Laryngologica, 120, 273-278. http://dx.doi.org/10.1080/000164800750001080

[13] Karakas, S. and Kavakli, A. (2005) Morphometric examination of the paranasal sinuses and mastoid air cells using computed tomography. Annals of Saudi Medicine, 25, 41-45.

[14] Kazkayasi, M., Karadeniz, Y. and Arikan, O.K. (2005) Anatomic variations of the sphenoid sinus on computed tomography. Rhinology, 43, 109-114.

[15] Hatipoglu, H.G., Cetin, M.A., Selvi, A., et al. (2009) Role of magnetic resonance imaging in evaluating sphenoid sinus and internal carotid artery. Journal of Laryngology \& Otology, 123, 1331-1337. http://dx.doi.org/10.1017/S0022215109991046
[16] Davoodi, M., Saki, N., Saki, G., et al. (2009) Anatomical variations of neurovascular structures adjacent sphenoid sinus by using CT scan. Pakistan Journal of Biological Sciences, 12, 522-525. http://dx.doi.org/10.3923/pjbs.2009.522.525

[17] Cho, J.H., Kim, J.K., Lee, J.G., et al. (2010) Sphenoid sinus pneumatization and its relation to bulging of surrounding neurovascular structures. Annals of Otology, Rhinology \& Laryngology, 119, 646-650.

[18] Chen, Y.L., Lee, L.A. and Lim, K.E. (2006) Surgical consideration to optic nerve protrusion according to sinus computed tomography. Otolaryngology-Head and Neck Surgery, 134, 499-501. http://dx.doi.org/10.1016/j.otohns.2005.10.036

[19] Heskova, G., Mellova, Y., Holomanova, A., et al. (2009) Assessment of the relation of the optic nerve to the posterior ethmoid and sphenoid sinuses by computed tomography. Biomedical papers of the Medical Faculty of Palacký University, Olomouc, Czech Republic, 153, 149152. 